\title{
Ueber das Ritter-Rollett'sche Phaenomen.
}

Von

\author{
Dr. med. H. Osswald, \\ Assistent am physiologischen Institut in Tübingen.
}

Im Anfang unseres Jahrhunderts beobachtete Ritter ${ }^{1}$ ) eine merkwürdige Erscheinung bei indirekter galvanischer Reizung des Froschschenkels. Er fand, dass eine Verschiedenheit in der Erregbarkeit der Beuge- und Streckmuskeln vorhanden sei; die ersteren seien leichter erregbar und stürben früher $a b$, die letzteren antworteten erst auf stärkere Reize, hielten aber auch um so länger aus. Er nahm deshalb „eine beschränkte, bedingte, endliche" Erregbarkeit, die der Beuger; und „eine unbeschränkte, unbedingte, unendliche", die der Strecker, an.

Diesen Angaben Ritter's wurde zunäebst von Pfaff ${ }^{2}$ ) widersprochen, welcher die Regelmässigkeit der Ritter'schen Erscheinung anzweifelte.

Später ist dann Du Bois-Reymond in seinem Werke

1) J. W. Ritter, Beiträge zur nähern Kenntniss des Galvanismus etc. 2. Band, 3., 4. und letztes Stück „Darstellung des Gegensatzes zwischen Flexoren und Fixtensoren und ihren Frregbarkeiten, wie ihn galvanische Versuche ergeben und Reduction derselben auf einen andern, neuen, überall verbreiteten Gegensatz." Jena 1805.

2) Pfaff, Scheel, Rudolphi, Nordisches Archiv für Naturkunde etc. 1805, Band IV, Stück 3, No. XII, pag. 3 und Gehler's Physikalisches Wörterbuch Band IV, Abth. 2, 1828 pag. 720. - Es lassen übrigens $P$ faff's Worte auf pag. 722 des letzten Werkes: „Darin stimmen meine Erfahrungen mit denen jenes Gelehrten überein, dass die Einwirkung des galvanischen Reizes auf die Flexoren nur auf der ersten, höchsten Stufe der Reizbarkeit stattfindet, dass diese gewohnlich schnell verschwindet und die Reizbarkeit der Extensoren nach sehr kurzer Zeit das Uebergewicht erhält" keinen Zweifel darüber obwalten, dass er das von Ritter beobachtete Phaenomen wirklich gesehen. 
„Ueber thierische Electricität" "1) des Näheren auf die Arbeiten Ritter's und anch auf dessen Angaben über die uns interessirende Erscheinung eingegangen. Er hält die Ritt e r'schen Lehren für sehr unwahrscheinlich und meint, man solle sie so lange für beseitigt ansehen, bis erneute, unzweideutige Untersuchungen darüber angestellt seien.

Dieser Aufgabe hat sich im Jahre 1874 Rollett ${ }^{2}$ ) unterzogen, welcher die Ritte r'schen Beobachtungen wieder der Vergessenheit entriss und sie durch zahlreiche, einwandsfreie Versuche bestätigte. Später hat dann Frl. Völk i n ${ }^{3}$ ) im Herman n'schen Laboratorium gefunden, dass der gleiche Unterschied in der Erregbarkeit der Beuger und Streeker auch beim Kaninchen vorhanden ist, wovon ich mich durch eigene Versuche überzengen konnte.

Um unsere Erscheinung hervorzurufen, wurde bisher nur die elektrische Reizung, sowohl der galvanische (Ritter); als auch der Inductionsstrom (Rollett), angewendet. Ich habe versucht, auch mittelst anderer, zunächst $\mathrm{chem}$ is cher Nerven reizmittel das Ritter-Rollett'sche Pbaenomen hervorzurnfen, was mir gelungen ist. Es sind, soweit mir bekannt geworden ist, derartige Versuche nur von Grützne ${ }^{4}$ ) frïher kurz erwähnt worden.

Als Versuchsobjekte benutzte ich bei der Mehrzahl der Versuche kräftige Wiesenfrösche (Rana temporaria), die theils im Institut überwintert hatten, theils frisch eingefangen waren, dann auch Kröten (Bufo vulgaris). Bei letzteren ist der Unterschied in der Eiregbarkeit der Beuger und Strecker überaus deutlich vorhanden. - Später wurden noch eine Anzahl Versuche mit grïnen Fröschen (Rana esculenta), die mir anfangs nicht zu Gebote standen, angestellt. Meine Versuche wurden rom März bis Mai dieses Jahres im hiesigen physiologisehen Institut unter Leitung des Herrn Prof. Dr. Grützner ausgeführt, dem ich für die Anregung zu

1) Du Bois-Reymond: Untersuchungen über thierische Electricität Band I, Berlin 1848, pag. 317 etc.

2) Rollett, Sitzungsberichte d. Wien. Acad. math.-naturw. Classe, Abth. 3, Band 70 pag. 7, Band 71 pag. 33, Band 72 pag. 349, 1874-76.

3) Hermann's Handbuch Band I, 1 pag. 113.

4) Zur Physiologie u. Histologie der Skelettmuskeln. Bresl. ärztl. Zeitschrift 1883, No. 24 pag. 257. 
dieser Arbeit und für seine freundliche Unterstiitzung bei derselben auch an dieser Stelle meinen herzlichsten Dank ausspreche.

Chemische $\operatorname{Reize}^{1}$ ).

Nachdem del Frosch (resp. die Kröte) getödtet und enthäntet, wurde der Ischiadicus bis zur Kniekehle freigelegt, der Oberschenkel von den Mnskeln befreit und hoch oben amputirt, der Nerv so rein als möglich präparirt und der Plexus dicht an der Wirbelsäule unterbunden und oberhalb der Ligatur abgeschnitten. Der auf diese Weise hergerichtete Unterschenkel wurde am Knochenstumpf des Femur in einer Klemme befestigt und in einem mit physiologischer Kochsalzlösung gefüllten Glaskasten mit ebenen Wänden aufgehängt, durch dessen Wände eine genaue Beobachtung des Präparats möglich war. Es besitzt die physiologische Kochsalzlösung annähernd das gleiche spezifische Gewicht wie der Froschschenkel. In Folge dessen wird letzterer nahezu schwerlos und es können deshalb schon die geringsten Contractionen der Muskeln eine deutlich sichtbare Wirkung hervorrufen. Es ist deshalb diese seiner Zeit von $G r$ iutzne ${ }^{2}$ ) angegebene Methode zu derartigen Untersuchungen, falls es sich um genaue Beobachtungen der Contractionen einzelner Muskeln oder Muskelantheile handelt, sehr zu empfehlen.

Dicht ỉber der Oberfläche des gefüllten Gefässes wurde ein kleines Uhrschälchen angebracht und mit der betreffenden Reizflüssigkeit, welche ebenso wie die $0,6 \%$ Kochsalzlösung stets die Temperatur der Umgebung, circa $18^{\circ} \mathrm{C}$. hatte, angefüllt und vom oberen Ende des Nerven eine 1-2 cm lange Strecke hineingelegt. Das nicht in der Reizflüssigkeit befindliche Stück des Nerven schwamm in der $0,6 \%$ Kochsalzlösung bis auf das kleine Endchen, welches von dem Rande des Glasschälchens bis zur Oberfläche des Flüssigkeitsspiegels reichte, wodurch eine Vertrocknung des Nerven ausgeschlossen wurde.

Ich beobachtete, $\mathrm{um}$ in Kürze die Erscheinungen, wie sie sich bei einem derartigen Versuche zeigten, zusammenzufassen, folgendes :

1) Verzeichnisse der Litteratur über chemische Nervenreizung siehe in Hermann's Handbuch Band II, pag. 96 und bei Limbourg, Beiträge zur chemischen Nervenreizung etc. Pflüger's Archiv Band 41 pag. 324.

2) Pflüger's Archiv Band 41, pag. 278. 
Nach dem Einlegen des Nerven in die Reizflüssigkeit beginnen nach kürzerer óder längerer Zeit, die von der Art und Concentration des Reizmittels und auch von der Art des Versuchsthieres (bei der Kröte nach läng'erer Zeit als beim Frosch) abhängt, Zuckungen in den Zehen und zwar fast konstant in den Extensoren der Zehen und den dorsalen Zwischenknochenmuskeln, bisweilen auch in den Zehenflexoren oder in beiden gleichzeitig, aber viel seltener. - Kurze Zeit darauf fangen die Motoren des Fusses nach vor- und aufwärts, (die den grossen, stumpfen Winkel, welchen der herabhängende Fuss im Fussgelenk bildet, verkleinern,) kurzweg $\mathrm{Beuger}^{1}$ ) genannt, an, in Wirksamkeit zu treten. Nachdem diese eine Weile ganz allein thätig gewesen, (besonders bei der Kröte ist dieses Stadium sehr ausgesprochen), beginnen auch Contractionen der Strecker ${ }^{2}$ ), namentlich des Gastrocnemius, welche zuerst noch nicht das Uebergewicht haben. Es tritt ein Stadium des Kampfes ein zwischen Bengung und Streckung, welehes gewöhnlich bald mit einem völligen Siege der Strecker und einem ausgesprochenen Strecktetanus von längerẻr. Dauer endigt.- Wirkt das angewandte Reizmittel sehr schädigend auf den Nerven ein, so kann ein ausgesprochener Tetanus entweder ganz fehlen oder nur von kurzer Dauer sein.

Was die im Anfang auftretenden Beugungen anlangt, so sind diese am ausgesprochensten bei der Kröte. Es tritt bei letzteren eine U-förmige Dorsalflexion ein, wie man sie beim Froscb in der Regel nur bei Reizung des Ischiadicus nach Durchschneidung des Nervus tibialis beobachtet and wie sie Rollett ${ }^{3}$ ) in seiner $\mathrm{Ab}$ handlung abbildet.

Als Reizmittel wurden die versehiedensten angewandt, bei der Mehrzahl der Versuche das Chlornatrium. Es erwiesen sich

1) Es gehören hierzu folgende Muskeln: der M. tibialis ant., M. peroneus, M. flexor tarsi ant. u. post., die M. abductores u. extensores digitorum und die M. interossei dorsales. Sie werden in der Hauptsache versorgt vom Nerv. peroneus.

2) Es gehören bierzu folgende Muskeln: der M. gastrocnemius, der M. tibialis post., der M. extensor tarsi, der M. plantaris, die M. transversi plantae, die $\mathbf{M}$. flexores digitorum, die M. lumbricales, die M. adductores digitorum, die M. interossei plantares. Sie werden vom Nerv. tibialis versorgt. In der Benennung der einzelnen Muskeln richtete ich mich nach Ecker, Anatomie des Frosches, Braunschweig 1864, pag. 119-137.

3) Sitzungsber. d. W. Acad., 3. Abth., Band 70, Heft 6, Taf. I, Fig. 2. 
davon Lösungen von $15: 100 \mathrm{Aq}$. destill. bis zur Sättigung (36:100) als tauglich, um auf das eklatanteste das Ritter-Rollett'sche Phaenomen hervorzurufen. Ich lasse hier einige Versuche folgen:

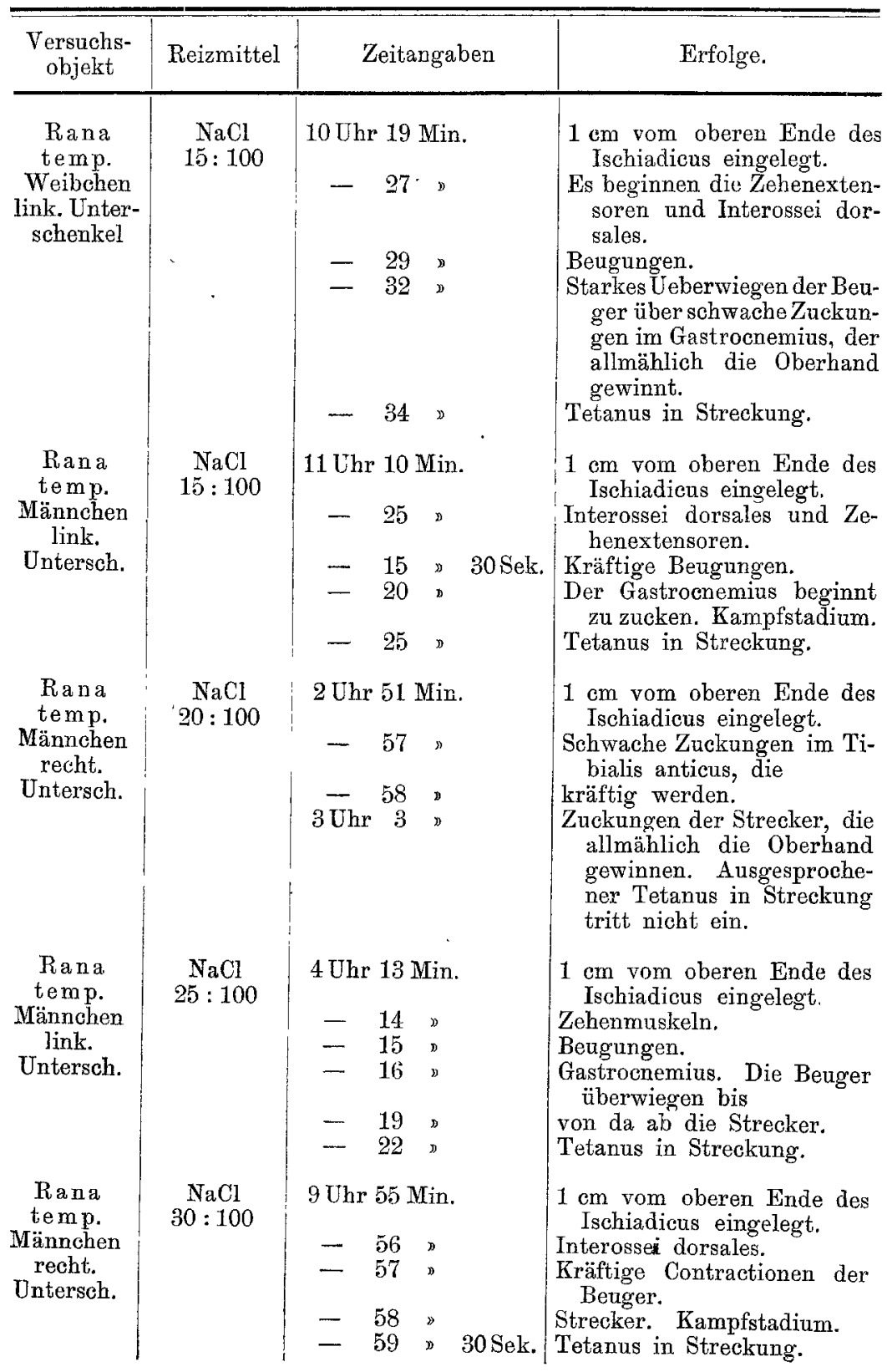




\begin{tabular}{|c|c|c|c|}
\hline $\begin{array}{l}\text { Versuchs- } \\
\text { objekt }\end{array}$ & Reizmittel & Zeitangaben & Erfolge. \\
\hline $\begin{array}{l}\text { Rana } \\
\text { temp. } \\
\text { Weibchen } \\
\text { recht. } \\
\text { Unterseh. }\end{array}$ & $\begin{array}{c}\mathrm{NaCl} \\
\text { gesättigte } \\
\text { Lösung }\end{array}$ & $\begin{array}{c}10 \text { Uhr } 31 \text { Min. } \\
-\quad 32 » \\
-\quad 33 \% \\
-\quad 34 \%\end{array}$ & $\begin{array}{l}1 \mathrm{~cm} \text { vom oberen Ende des } \\
\text { Ischiadicus eingelegt. } \\
\text { Es. beginnen sogleich Beu- } \\
\text { gungen. } \\
\text { Streckungen. } \\
\text { Tetanus in Streckung. }\end{array}$ \\
\hline $\begin{array}{l}\text { Rana } \\
\text { esculenta } \\
\text { Männchen } \\
\text { link. } \\
\text { Untersch. }\end{array}$ & $\begin{array}{c}\mathrm{NaCl} \\
30: 100\end{array}$ & $\begin{array}{l}9 \text { Uhr } 34 \text { Min. } \\
-\quad 38 \% 30 \text { Sek. } \\
-\quad 39 » \\
-\quad 42 ” \\
-\quad 44 \%\end{array}$ & $\begin{array}{l}1 \mathrm{~cm} \text { vom oberen Ende des } \\
\text { Ischiadicus eingelegt. } \\
\text { Zehenextensoren und Inter- } \\
\text { ossei dorsales. } \\
\text { Sehr kräftige Beugungen. } \\
\text { Erste Zuckungen im Gastro- } \\
\text { cnemius. Kampfstadium. } \\
\text { Uebergewicht der Strecker. } \\
\text { Kein ausgesprochener } \\
\text { Strecktetanus. }\end{array}$ \\
\hline $\begin{array}{l}\text { Bufo } \\
\text { vulgaris } \\
\text { Männchen } \\
\text { recht. } \\
\text { Untersch. }\end{array}$ & $\begin{array}{c}\mathrm{NaCl} \\
30: 100\end{array}$ & $\begin{array}{l}3 \text { Uhr } 56 \text { Min. } \\
4 \quad 11 \\
=13 \\
=\quad 16 \\
-\quad 21\end{array}$ & $\begin{array}{l}2 \mathrm{~cm} \text { vom oberen Ende des } \\
\text { Ischiadicus eingelegt. } \\
\text { Leise Zuckungen im Tibialis } \\
\text { anticus. } \\
\text { Ausgesprochene Beugungen. } \\
\text { Strecker. Kampfstadium. } \\
\text { Tetanus in Streckung. }\end{array}$ \\
\hline $\begin{array}{l}\text { Bufo } \\
\text { vulgaris } \\
\text { Männchen } \\
\text { recht. } \\
\text { Untersch. }\end{array}$ & $\begin{array}{c}\mathrm{NaCl} \\
30: 100\end{array}$ & $\begin{array}{l}3 \text { Uhr } 39 \text { Min. } \\
-\quad 49 \\
-55 \\
-\quad 56 \\
4 \text { Uhr } 1\end{array}$ & $\begin{array}{l}\text { 1,5 cm vom oberen Ende des } \\
\text { Ischiadicus eingelegt. } \\
\text { Sehr starke Beugungen, die } \\
\text { bis } \\
\text { anhalten. } \\
\text { Gastrocnemius. Kampfsta- } \\
\text { dium. } \\
\text { Tetanus in Streckung. }\end{array}$ \\
\hline
\end{tabular}

Ferner wurden angewandt das Fluornatrium in gesättigter Lösung (4:100 Aq. dest.), das Bromnatrium und Jodnatrium in den verschiedensten Concentrationen. Alle riefen deutlich unsere Erscbeinung hervor. Am stärksten schädigend wirkte das Jodnatrium auf den Nerven ein, so dass die Erregbarkeit desselben schon nach kurzer Zeit verniehtet wurde. Ich führe wiederum die Ergebnisse einzelner Versuche an: 


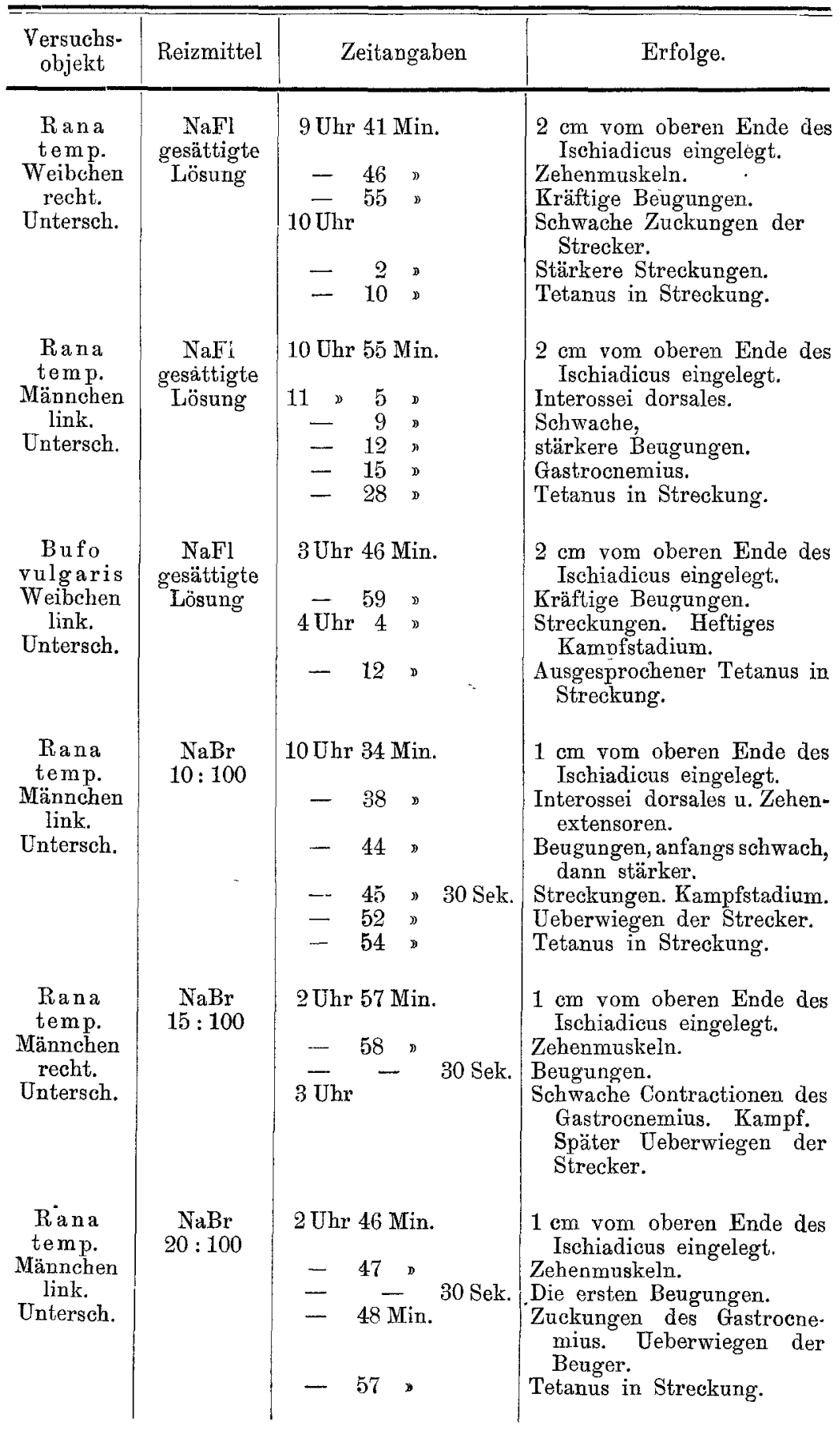




\begin{tabular}{|c|c|c|c|}
\hline $\begin{array}{l}\text { Versuchs- } \\
\text { objekt }\end{array}$ & Reizmittel & Zeitangaben & Erfolge. \\
\hline $\begin{array}{c}\text { Rana } \\
\text { temp. } \\
\text { Weibchen } \\
\text { link. } \\
\text { Untersch. }\end{array}$ & $\begin{array}{c}\mathrm{NaBr} \\
50: 100\end{array}$ & $\begin{array}{l}10 \text { Uhr } 16 \text { Min. } \\
-\quad 17 \\
=21 \\
=24 \\
-\quad 46\end{array}$ & $\begin{array}{l}2 \mathrm{~cm} \text { vom oberen Ende des } \\
\text { Ischiadicus eingelegt. } \\
\text { Zehenextensoren und Inter- } \\
\text { ossei dorsales. } \\
\text { Sehr schöne Beugungen. } \\
\text { Vereinzelte Contractionen } \\
\text { des Gastrocnemius. Kampf- } \\
\text { stadium. } \\
\text { Ueberwiegen der Strecker. } \\
\text { Tetanus in Streckung. }\end{array}$ \\
\hline $\begin{array}{c}\text { Bufo } \\
\text { vulgaris } \\
\text { Weibchen } \\
\text { link. } \\
\text { Untersch. }\end{array}$ & $\begin{array}{c}\mathrm{NaBr} \\
20: 100\end{array}$ & $\begin{array}{l}4 \text { Uhr } 21 \text { Min. } \\
-\quad 27 " \\
-\quad 29 " \\
-\quad 30 \% \\
-\quad 32 》\end{array}$ & $\begin{array}{l}2 \mathrm{~cm} \text { vom oberen Ende des } \\
\text { Ischiadicus eingelegt. } \\
\text { Sehr starke Beugungen. } \\
\text { Schwache, } \\
\text { stärkere Contractionen des } \\
\text { Gastrocnemius. Kampfsta- } \\
\text { dium. } \\
\text { Tetanus in Streckung. }\end{array}$ \\
\hline $\begin{array}{l}\text { Rana } \\
\text { temp. } \\
\text { Männchen } \\
\text { link. } \\
\text { Untersch. }\end{array}$ & $\begin{array}{c}\text { NaJ } \\
10: 100\end{array}$ & $\begin{array}{l}11 \text { Uhr } 21 \text { Min. } \\
-43 \\
-45\end{array}$ & $\begin{array}{l}\text { 1,5 cm vom oberen Ende des } \\
\text { Ischiadicus eingelegt. } \\
\text { Es beginnen die Beuger, } \\
\text { die Strecker zu wirken. } \\
\text { Kein Tetanus. }\end{array}$ \\
\hline $\begin{array}{l}\text { Rana } \\
\text { temp. } \\
\text { Männchen } \\
\text { recht. } \\
\text { Untersch. }\end{array}$ & $\begin{array}{c}\mathrm{NaJ} \\
20: 100\end{array}$ & $\begin{array}{l}10 \text { Chr } 26 \text { Min. } \\
-\quad 29 \\
-\quad 31\end{array}$ & $\begin{array}{l}1,5 \mathrm{~cm} \text { vom oberen Fnde des } \\
\text { Ischiadicus eingelegt. } \\
\text { Zehenextensoren u. Beuger. } \\
\text { Streckungen. Kampfstadium. } \\
\text { Schliesslich kurz dauern- } \\
\text { der Tetanus in Streckung. }\end{array}$ \\
\hline $\begin{array}{l}\text { Rana } \\
\text { temp. } \\
\text { Männchen } \\
\text { recht. } \\
\text { Untersch. }\end{array}$ & $\begin{array}{c}\mathrm{NaJ} \\
50: 100\end{array}$ & $\begin{array}{l}5 \text { Uhr } 1 \text { Min. } \\
-\quad 3 \% \\
=\quad 5 \% \\
=\quad 15 \%\end{array}$ & $\begin{array}{l}1 \text { am vom oberen Ende des } \\
\text { Ischiadicus eingelegt. } \\
\text { Beugungen. } \\
\text { Streckungen. } \\
\text { Aufhören der Zuckungen. } \\
\text { Nerv im Bereich der einge- } \\
\text { legten Strecke elektrisch } \\
\text { nicht mehr erregbar. }\end{array}$ \\
\hline $\begin{array}{c}\text { Rana } \\
\text { temp. } \\
\text { Weibchen } \\
\text { link. } \\
\text { Untersch. }\end{array}$ & $\begin{array}{c}\mathrm{NaJ} \\
75: 100\end{array}$ & 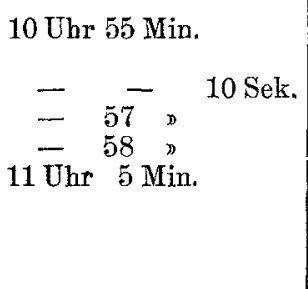 & $\begin{array}{l}1,5 \mathrm{~cm} \text { vom oberen Ende des } \\
\text { Ischiadicus eingelegt. } \\
\text { Beugungen. } \\
\text { Gastrocnemius. Kampf. } \\
\text { Aufhören der Zuckungen. } \\
\text { Der Nerv im Bereich der } \\
\text { eingelegten Strecke elek- } \\
\text { trisch nicht mehr erreg- } \\
\text { bar. }\end{array}$ \\
\hline
\end{tabular}


Ferner wurden angewendet Lösungen von Chlor-, Brom- und Jodkalium in verschiedenen Concentrationen. Die beiden letzteren Mittel erregten überhaupt keine Zuckungen; Chlorkalium bewirkte bei schwächeren Lösungen höchstens Contractionen der Zehenmuskeln, meistens traten ïberhaupt keine Zuckungen ein ${ }^{1}$ ). Mit gesättigter Lösung erhielt ich einige brauchbare Resultate, wovon ich folgendes Beispiel anfiibre:

\begin{tabular}{|c|c|c|c|}
\hline $\begin{array}{l}\text { Versuchs- } \\
\text { objekt }\end{array}$ & Reizmittel & Zeitangaben & Erfolge. \\
\hline $\begin{array}{l}\text { Rana } \\
\text { temp. } \\
\text { Männchen } \\
\text { link. } \\
\text { Untersch. }\end{array}$ & $\begin{array}{c}\text { KCl } \\
\text { gesästigte } \\
\text { Lösung }\end{array}$ & $\begin{array}{l}12 \text { Uhr } 6 \text { Min. } \\
-\quad 7 \% \\
=\quad 8 \% \\
-\quad 10 \% \\
-\quad 14 \%\end{array}$ & $\begin{array}{l}1 \mathrm{~cm} \text { vom oberen Ende des } \\
\quad \text { Ischiadicus eingelegt. } \\
\text { Zehenextensoren. } \\
\text { Beuger. } \\
\text { Strecker. } \\
\text { Tetanus in Streckung. }\end{array}$ \\
\hline
\end{tabular}

Von Baryumsalzen hatte Baryum nitricum weder beim Frosch noch bei der Kröte Zuckungen zur Folge.

Chlorbaryum bewirkte in gesättigter Lösung verwendet das Ritter-Rollett'sche Phaenomen beim Frosch. Bei der Kröte waren nach 30 Minuten noch keine Zuckungen eingetreten.

\begin{tabular}{|c|c|c|c|}
\hline $\begin{array}{c}\text { Versuchs- } \\
\text { objekt. }\end{array}$ & Reizmittel & Zeitangaben & Erfolge. \\
\hline $\begin{array}{c}\text { Rana } \\
\text { temp. } \\
\text { Weibchen } \\
\text { link. } \\
\text { Untersch. }\end{array}$ & $\underset{\text { Lesättigte }}{\mathrm{BaCl}_{2}}$ & $\begin{array}{l}3 \text { Uhr } 9 \text { Min. } \\
-\quad 14 ” \\
-\quad 17 \% \\
=\quad 19 \% \\
-\quad 21 \% \\
-\quad 26 »\end{array}$ & $\begin{array}{l}1 \text { cm vom oberen Ende des } \\
\text { Ischiadicus eingelegt. } \\
\text { Zehenextensoren und Inter- } \\
\text { ossei dorsales. } \\
\text { Sohwache, } \\
\text { kräftige Beugungen. } \\
\text { Streckungen. } \\
\text { Ausgesprochener Tetanus in } \\
\text { Streckung. }\end{array}$ \\
\hline $\begin{array}{l}\text { Rana } \\
\text { tèmp. } \\
\text { Männchen } \\
\text { recht. } \\
\text { Untersch. }\end{array}$ & $\begin{array}{c}\mathrm{BaCl}_{2} \\
\text { gesättigte } \\
\text { Lösung }\end{array}$ & $\begin{array}{l}9 \text { Uhr } 24 \text { Min. } \\
-\quad 27 \\
-\quad 32 \\
-\quad 33 \\
-\quad 40\end{array}$ & $\begin{array}{l}\text { 1,5 cm vom oberen Ende des } \\
\text { Ischiadicus eingelegt. } \\
\text { Interossei dorsales. } \\
\text { Beugungen. } \\
\text { Streckungen. } \\
\text { Tetanus in Streckung. }\end{array}$ \\
\hline
\end{tabular}

1) Auf die schädigende Wirkung der Kalisalze auf die Nerven hat Grützner auf der Bremer Naturforscherversammlung 1890 (Berichte derselben, Theil II, pag. 177) aufmerksam gemacht. 
Von vorzüglicher Wirkung als Nervenreizmittel ist auch das Glycerin. Es wurde unverdiunt und 10:1 Aq. dest. angewendet. Die Resultate einiger Versuche sind z. B. folgende:

\begin{tabular}{|c|c|c|c|}
\hline $\begin{array}{c}\text { Versuchs- } \\
\text { objekt }\end{array}$ & Reizmittel & Zeitangaben & Erfolge. \\
\hline $\begin{array}{l}\text { Rana } \\
\text { temp. } \\
\text { Weibohen } \\
\text { recht. } \\
\text { Untersch. }\end{array}$ & $\begin{array}{c}\text { Glycerin } \\
\text { unverdünnt }\end{array}$ & $\begin{array}{l}\text { 10 Uhr } 26 \text { Min. } \\
-27 \\
=\quad 28 \text { Min. } 30 \text { Sek. }\end{array}$ & $\begin{array}{l}1,5 \mathrm{~cm} \text { vom oberen Ende des } \\
\text { Ischiadicus eingelegt. } \\
\text { Zehenmuskeln. Beugungen. } \\
\text { Streckungen. Kampfstadium. } \\
\text { Tetanus in Streckung. }\end{array}$ \\
\hline $\begin{array}{l}\text { Rana } \\
\text { temp. } \\
\text { Weibchen } \\
\text { recht. } \\
\text { Untersch. }\end{array}$ & $\begin{array}{c}\text { Glycerin } \\
10: 1 \text { Aq. } \\
\text { dest. }\end{array}$ & \begin{tabular}{|l}
11 Uhr 22 Min. \\
$-\quad 26 \succsim$ \\
$-\quad 30$ \\
$-\quad 32$ \\
$-\quad 35$
\end{tabular} & $\begin{array}{l}1 \text { cm vom oberen Ende des } \\
\text { Ischiadicus eingelegt. } \\
\text { Zehenmuskeln. } \\
\text { Beugungen. } \\
\text { Streckungen. Kampf. } \\
\text { Tetanus in Streckung. }\end{array}$ \\
\hline $\begin{array}{l}\text { Bufo } \\
\text { vulgaris } \\
\text { Weibchen } \\
\text { link. } \\
\text { Intersch. }\end{array}$ & $\begin{array}{c}\text { Glycerin } \\
\text { unverdünnt }\end{array}$ & $\begin{array}{c}10 \text { Uhr } 4 \text { Min. } \\
-\quad 18 \% \\
-\quad 19 \% \\
-\quad 20 \%\end{array}$ & $\begin{array}{l}\text { 1,5 cm vom oberen Ende des } \\
\text { Ischiadicus eingelegt. } \\
\text { Zehenextensoren und Inter- } \\
\text { ossei dorsales. } \\
\text { Starke Beugungen. } \\
\text { Gastrocnemius. Kampfsta- } \\
\text { dium. } \\
\text { Tetanus in Streckung. }\end{array}$ \\
\hline $\begin{array}{l}\text { Bufo } \\
\text { vulgaris } \\
\text { Weibchen } \\
\text { link. } \\
\text { Untersch. }\end{array}$ & $\begin{array}{c}\text { Glycerin } \\
10: 1 \mathrm{Aq} \text {. } \\
\text { dest. }\end{array}$ & $\begin{array}{l}2 \text { Uhr } 59 \text { Min. } \\
3 \text { Uhr } 19 \text { Min. } \\
-23 \\
-\quad 24 \\
-\quad 26\end{array}$ & $\begin{array}{l}2 \text { cm vom oberen Ende des } \\
\text { Ischiadicus eingelegt. } \\
\text { Hochgradige Bengungen, } \\
\text { welche bis } \\
\text { andauern. } \\
\text { Zuckungen des Gastrocne- } \\
\text { mius. Kurzer Kampf. } \\
\text { Tetanus in Streckung. }\end{array}$ \\
\hline $\begin{array}{c}\text { Bufo } \\
\text { vulgaris } \\
\text { Männchen } \\
\text { link. } \\
\text { Untersch. }\end{array}$ & $\begin{array}{c}\text { Glycerin } \\
10: 1 \mathrm{Aq} . \\
\text { dest. }\end{array}$ & $\begin{array}{r}11 \text { Uhr } 20 \text { Min. } \\
=\quad 38 \\
=\quad 40 \\
-\quad 50\end{array}$ & $\begin{array}{l}1,5 \mathrm{~cm} \text { vom oberen Ende des } \\
\text { Ischiadicus eingelegt. } \\
\text { Interossei dorsales. } \\
\text { Kräftige Beugungen. } \\
\text { Schwache Zuckungen des } \\
\text { Gastrocnemius, die all- } \\
\text { mählich zunehmen. Kampf. } \\
\text { Tetanus in Streckung. }\end{array}$ \\
\hline
\end{tabular}

Die Ergebnisse dieser Versuche, von denen ich eine Anzahl in deu vorhergehenden Tabellen mitgetheilt habe, zeigen aufs deutlichste, dass auch chemische Agentien als Nervenreizmittel angewandt im Stande sind, am Unterschenkel von Fröschen und Kröten den Nervmuskelapparat der 
Beuger früher zu erregen als dender Strecker, also das sog. Ritter-Rollet'sche Phaenomen hervorzurufen.

Da nach der gewöhnlichen Annahme die Wirksamkeit vieler chemischer Nervenreizmittel auf der Was s e e $\mathrm{ntziebung}$ beruht, lag der Gedanke nahe, zu untersuchen, ob sich der Unterschied in der Erregbarkeit der Beuger und Strecker auch be i e in facher Vertrocknung des Nerven zeige. Es gelangen diese Versuche besonders gut bei der Kröte, aber auch bei Fröschen (Rana temporaria und esculenta). Ich stellte die Versuche in folgender Weise an:

Der wie erwähnt präparirte Unterschenkel wird frei in einer Klemme befestigt. Sein Nerv, den man zwischen Filtrirpapier sorgfältig abgetrocknet und mittelst des zu seiner Abbindung an ihm befindlichen Fadens in wagerechter Richtung lose aufgehängt hat, wird dem Vertrocknen überlassen, was bei einigen Versuchen durch ein darunter befindliches Gefäss mit concentrirter Schwefelsäure ein wenig beschleunigt werden konnte. - Das Vertrocknen der Muskeln wurde durch häufiges Beträufeln mit physiologischer Kochsalzlösung verhindert. Ich fïhre einige derartige Versuche an:

Bufo vulgaris, Weibchen, linker Unterschenkel. Der Nerv wird 9 Uhr 39 Min. abgetrocknet und aufgehängt. $10 \mathrm{Uhr} 9 \mathrm{Min}$. treten sehr deutliche Beugungen auf, welche bis $10 \mathrm{Uhr} 12 \mathrm{Min}$. anhalten. Jetzt beginnt der Tibialis posticus und Plantaris und $10 \mathrm{Jhr} 13 \mathrm{Min}$. der Gastrocnemius. Heftiger Kampf zwischen Beugung und Streckung, der 10 Uhr 17 Min. mit Tetanus in Streckung endet.

Bei der Kröte fielen alle Versuche derartig aus. Bei den männlichen Fröschen zeigte sich das gleiche Verhalten, z. B. bei folgenden Versuchen:

Rana temporaria, Männehen, linker Unterschenkel. Der Nerv wird abgetrocknet und $9 \mathrm{Uhr} 43 \mathrm{Min}$. über concentrirter Schwefelsäure aufgehängt. 9 Uhr 47 Min. beginnen die ersten Zuckungen in den Beugern ohne deutliche Wirkung. 9 Uhr 54 Min. werden die Contractionen der Beuger kräftig, zugleich treten auch solche des Gastrocnemius auf. Die Beuger überwiegen, bis $9 \mathrm{Uhr} 55 \mathrm{Min}$. ausgesprochener Tetanus in Strecknng eintritt.

R ana esculenta, Männchen, rechter Unterschenkel. Der Nerv wird $3 \mathrm{Uhr} 54$ Min. abgetrocknet und aufgehängt. $3 \mathrm{Uhr} 56 \mathrm{Min}$. beginnen bereits deutliche Beugungen. $3 \mathrm{Uhr} 58$ Min. beginnt auch der Gastrocnemius zu zucken, doch überwiegen noch die Beuger. Von $4 \mathrm{Uhr}$ ab erhalten die Strecker das Uebergewicht. Tetanus. 
Bei den weiblichen Fröschen wurden bei derartigen Versuchen dentliche Beugungen nicht beobachtet. Es überwogen sehr frïh die Streckungen.

Rana esculenta, Weibchen, rechter Unterschenkel. Der Nerv wird $11 \mathrm{Uhr} 35 \mathrm{Min}$. abgetrocknet und aufgehängt. $11 \mathrm{Uhr} 39 \mathrm{Min}$. bemerkt man fibrilläre Zuckungen der Beuger, welcher aber keine deutlichen Beugungen hervorriefen. Ganz kurze Zeit später beginnen Contractionen des Gastrocnemius, die aber eine völlige Streckung des Schenkels erst 11 Uhr 40 Min. zur Folge haben. 11 Uhr 41 Min. tritt ausgesprochener Tetanus in Streckung ein.

\section{Mechanische Reize ${ }^{\mathbf{1}}$.}

Meine nächste Aufgabe war, zu versuchen, ob man dureh mechanische Nervenreizung das Ritter-Rollett'sche Phaenomen hervorrufen könne. Es kam mir darauf an, die mechanischen Reize sowohl hinsichtlich ihrer Zahl als auch ibrer Stärke möglichst bequem reguliren zu können. Ich bediente mich dazu eines Verfahrens, wie es zuerst $\mathrm{H}$ e i d e n ha in $\mathbf{1 8 5 6}$ in ähnlicher Weise benutzte. Ieh brachte am Anker eines W a g n e r'schen Hammers mit $\mathrm{H}$ elm hol tz'scher Modifikation ein kleines Elfenbeinhämmerchen an, welches an der Fläche, mit welcher es aufschlagen sollte, sorgfältig abgerundet war, um Schneidewirkungen zu vermeiden. Dieses Hämmerchen schlug auf eine quadratische elfenbeinerne Platte, welche an einem Stativ befestigt war. In diese Platte war eine Rinne eingeschliffen, weit genug, um den Ischiadicus eines Frosches bequem aufnehmen zu können. Da nun der Hammer so den in der Rinne befindlichen Nerven nicht hätte treffen können, wurde eine zweite $3 \mathrm{~mm}$ breite Rinne eingefeilt, welche die erste rechtwinklig schnitt und genau dieselbe Tiefe besass. Es konnten also die Schläge des Hammers auf den Nerven vollkommen einwirken, zugleich wurde dadurch eine Verschiebung des letzteren verhindert.

1) Aus der Litteratur erwähne ich: R. Heidenhaim, Physiologische Studien, pag. 132, 1856. - R. Tigerstedt, Studien über mechanische Nervenreizung. Helsingfors 1880 . $-\mathrm{R}$. Tig ersted t, Zeitschrift für Instrumentenkunde, Apparat für Nervenreizung, 1884, pag. 77. - Hälls tén, Zur Kenntniss der mechanischen Reizung der Nerven, Du Bois' Archiv 1881. Zederbaum, Nervendehnung und Nervendruck, Du Bois' Archiv 1883. Efron, Beiträge zur allgemeinen Nervenphysiologie, Pflüger's Archiv, Band 36, 1885. 
In den mit der beschriebenen Schlagvorrichtung versehenen Wa g n e r'schen Hammer mit $\mathrm{H}$ e $\mathrm{l}$ m h o l t z'seher Einrichtung wurde an Stelle der primären Rolle ein S i e m e n s'scher Stöpselrheostat eingeschaltet, der Hammer selbst in Bewegung gesetzt durch ein Da n i e 11 - Element von mittlerer Grösse oder 1-2 kleine G r ove-Elemente. Indem ich in den Rheostaten verschieden grosse Widerstände einschaltete und zugleich die am Wa $\mathrm{g} n$ er'schen Hammer angebrachte Feder stärker oder schwächer anspannte, konnte ich nach Belieben die Schläge des Hämmerchens aufs genaueste an Zahl und Intensität verändern. Auf diese Weise ist es mir gelungen, das Ritte $\mathrm{r}$ - R ollett'sche Phaenomen hervorzurufen. Als Versuchsobjekte benutzte ich zunächst Kröten und und dann Wasserfrösche.

Der Unterschenkel wurde genau so wie zu den chemischen Versuchen hergerichtet. Doch wurde er, um leichter mit ihm resp. dem Nerven Ortsveränderungen vornehmen zu können, nicht in physiologischer Kochsalzlösung aufgehängt, aber Nerv und Schenkel häufig mit ihr beträufelt, um Eintrocknung zu vermeiden.

Wurden nun die Schläge des Hämmerchens auf die oben beschriebene Weise möglichst abgeschwächt und wirkten sie dann auf den Nerven ein, so traten sehr deutliche Beugungen auf, die bei allmählicher Verstärkung der Reize mit Streckungen abwechselten; schliesslich überwogen völlig die Strecker und es trat Tetanus in Streckung ein, kurz ganz dasselbe Bild, wie es bei elektriseher und chemiseher Nervenreizung sich zeigt.

Es traten die Beugungen, um ein Beispiel anzuführen, an dem rechten Unterschenkel einer weiblichen $R$ a n a es culenta anf (bei $1 \mathrm{D}$ an i ell-Element) bei einem Widerstand von $3 \mathrm{~S}$ i e nens und mässig starker Federspannung, wobei der Hammer 8-10 Schläge in der Sekunde auf den Nerven ausübte. - Bei den Kröten konnten stärkere Schläge angewendet werden. Verschiedene Stellen der Nerven ergaben meist das gleiche Resultat, doch brauchten bisweilen die untern, den Muskeln benachbarten Nervenabschnitte stärkere Schläge, damit Zuckungen auftraten, als die oberen (wie auch Efron ${ }^{1}$ ) gefunden), worauf jedoch näher einzugehen hier nicht der Ort ist.

1) Efron, op. cit. pag. 496, vergl. dagegen Tigerstedt, Studien über mech. Nervenreizung p. 55 etc. 
Worauf beruht der Untersehied in der Erregbarkeit der Beuger und Strecker?

Nachdem im vorgergehenden Theil dieser Arbeit gezeigt worden, dass die Beuger des Unterschenkels bei Fröschen und Kröten nicht allein elektrischen, sondern auch chemischen und mechanischen Nervenreizen gegenüber leichter und frïher erregbar sind, als die Strecker, so diurfte es von Interesse sein, im Folgenden einzugehen auf die mannichfachen Erklärungen, die man seit dem Erscheinen der Rollett'schen Arbeiten für unser Phaenomen zu geben versucht hat.

R o l l e t t selbst meint, dass, „da bei direkter Reizung der Wuskeln der Unterschied nicht hervortritt, die Erklärung der Erscheinung im Nerven zu suchen ist" ${ }^{4}$ ).

Im Gegensatz zu ihm hat Bo $\mathrm{ur}^{2}$ ) in seiner unter Fick's Leitung verfassten Dissertation sich bemüht, unsre Erscheinung einfach so zu erklären, dass bei hängender, also nahezu gestreckter Lage des Froschschenkels Beugung leichter sichtbar sei als Streckung. Man beobachtet aber unsre Erscheinung durchans nicht blos bei hängender Lage des Froschschenkels, und Rollett konnte mittelst seines Antagonistographen nachweisen, dass bei Erregung vom Nerven aus die Beuger auf schwächere Reize grössere Arbeit leisten als die Strecker $\left.{ }^{3}\right)$. Ferner weisen nicht blos Beuger und Strecker des Unterschenkels Verschiedenheiten in der Erregbarkeit auf, sondern am Obersehenkel ist z. B. die Vastusgruppe viel erregbarer als die Bicepsgruppe, wie Frl. Neu$\operatorname{man}^{4}$ ) gefunden und wovon man sich leicht überzengen kann. Aehnliche Verhältnisse sind auch an der oberen Extremität vorbanden bei Fröschen und Kröten. Es bedingt hier das Geschlecht einen wesentlichen Unterschied. Beim Weibchen tritt bei Reizung: des Plexus brachialis mit allmählich stärker werdenden Induktionsströmen zuerst Beugung des Armes und dann Streckung ein. Beim Männchen dagegen zuerst Streckung und dann Beugung, welch'

1) Sitzungsberichte 3. Abth., Band 72, pag. 390 .

2) Bour, Ueber die verschiedene Erregbarkeit functionell verschiedener Nervmuskelapparate. Inaug.-Diss. Würzburg 1875.

3) Sitzungsberichte Band 72, pag. 379-386 und pag. 389.

4) Ueber toxicologische Verschiedenheiten functionell versehiedener Muskelgsuppen. Inaug.-Diss. Bern 1883. 
letztere der sehr kräftige Umarmungsmuskel (M. flexor carpi I s. Radialis (E c ker)) bewirkt. Ich fand dies Verhalten konstant bei einer Anzahl von Versuchen, die ich anstellte, und Herr Prof. Gr ï tz n er theilte mir mit, dass er früher bei den gleichen Versuchen dieselben Resultate, die ich mitgetheilt, erhalten habe.

Ferner hat man an ganz andern Nervmuskelapparaten analoge Verhältnisse beobachtet.

So baben $R$ ie he ${ }^{1}$ ) und $L u c h s i n g e r{ }^{2}$ ) gefunden, dass die Krebsscheere bei schwachen Reizen sich öffnet, bei starken schliesst, und Grü tzn e ${ }^{8}$ ) fand, dass bei Reizung des Vagus mit schwachen Strömen sich wesentlich die Verengerer der Stimmritze, bei starker Reizung regelmässig die Erweiterer derselben kontrahiren, und dass auch die vom Facialis versorgten Muskelgruppen Verschiedenheiten zeigen.

H. $\mathrm{MuK}^{4}$ ) hat versucht, dadurch unsere Erscheinung zu erklären, dass die einzelnen Nervenfasern ungleiche Stromzweige erhielten. Doch ist diese Erklärung desshalb nicht zulässig, weil man die Lage des Nerven zu den Elektroden beliebig verändern und anch ringförmige Elektroden benatzen kann 5 ), ohne irgendwie abweichende Resultate zu bekommen.

Hermann ${ }^{6}$ ) sagt: „Die Erscheinung tritt nur bei indirekter Reizung ein, muss also in Verhältnissen der Nervenfaser oder wahrscheinlich ihrer Endigung in den Muskeln begründet sein."

We de n's k $\mathrm{y}^{7}$ ) meint, dass ,das Wesen des Ritter-Rol1 e tl'schen Phaenomens auf dem verschiedenen Verhalten der antagonistischen Muskelgruppen zu der Reizfrequenz beruhe", d. b. dass bei maximalen Strömen mit wenigen Unterbrechungen Beugung, mit zahlreichen Streckung überwiege. Ich möchte dabei darauf hinweisen, dass bei wenigen Unterbrechungen die Reize doch wohl schwächer werden, da eine Summation dann viel weniger stattfinden kann. Es dürfte sich also wohl um ähnliche Verbältpag. 274.

1) Richet, Physiologie générale des muscles et des nerfs. Paris 1882.

2) Luchsinger, Pflüger's Archiv Band 28, pag. 60.

3) Breslauer ärztliche Zeitschrift 1883, pag. 190.

4) Reichert und Du Bois' Archiv 1875, pag. 41.

5) Rollett, Sitzungsberichte band 72, pag. 386-389.

6) Hermann's Handbuch Band I, 1, pag. 112.

i) Centralblatt für Physiologie Band I, 1888, pag. 272. 
nisse handeln wie bei gewöhnlicher Reizung mit schwachen Strömen und zahlreichen Unterbrechungen. Auch nach Wedensky kommt die Erscheinung nur bei indirekter Reizung zu Stande.

Ausser Bour sind alle erwähnten Autoren der Ansicht, dass es sich bei unserer Erscheinung um einen eigenthümlichen Zustand des Nerven resp. seiner Endigung in den Muskeln handle. Diese Annahme hatte auch ihre volle Berechtigung, so lange es nicht gelungen war, bei direkter Reizung der Muskeln unsere Erscheinung hervorzurufen.

Ich glaube dagegen mich dahin aussprechen zu dürfen, dass das Ritter-Rollett'sche Phaenomen berubtauch aufeiner physiologischen Verschiedenheit der Muskelgruppen der Beuger und Strecker, wahrscheinlich des gesammten Nerv-Muskelapparates beider. Dass die verschiedene Erregbarkeit in unserem Falle eine Eigenschaft der Muskelsubstanz sei, hat zuerst $G r$ ü $t \mathbf{z}$ e $\mathbf{r}^{1}$ ) ausgesprochen. Es liegt dieser Gedanke nahe, da wir ja wissen, dass durchaus nicht alle Muskeln gleichwerthig sind (ich erinnere an die rothen und weissen Muskeln des Kaninchen).

Es zeigt auch das Verhalten der Beuger und Strecker mannichfache Unterschiede, die man anf die Muskeln zurückführen muss. Die Beuger ermüden leichter als die Strecker (schon von Ritter beobachtet), sind ïberhaupt gegen Schädlichkeiten jeder Art viel empfindlicher. Nach Unterbindung der Iliaca auf einer Seite bemerkt man, dass schon nach kurzer Zeit nach dem Abspringen das kranke Bein langsamer angezogen wird als das gesunde, wie $G r \ddot{u} t z n \in r^{2}$ ) beobachtete, und wovon ich mich durch eigene Versuche überzeugte. Die Zuckungsgeschwindigkeiten der Flexoren infolge elektrischer Reize sind, ganz normale Erregbarkeitsverbältnisse vorausgesetzt, grösser als die der Extensoren $\left.(\text { Grïtzner })^{3}\right)$. Ferner ist der Tetanus der Beuger in der Regel „ein diskontinuirlicher, klonischer", der der Strecker „ein kontinuirlicher, tonischer"4), wovon ich mich auch überzeugen konnte,

1) Breslauer ärztliche Zeitschrift 1883, pag. 189.

2) Breslauer ärztliche Zeitschrift 1883, pag. 190.

3) Ebenda pag. 189. Waren die Erregbarkeitsverhältnisse nicht mehr normal, so konnte man auch das entgegengesetzte Verhalten beobachten.

4) Ebenda pag. 189. 
wenn ich die Ischiadici eines Frosches chemisch reizte, nachdem auf der einen Seite der Nervus tibialis, auf der andern der Nervus peroneus durchsehnitten war. Der Tetanus der Beuger zeigte beim Frosche meist das erwähnte Verhalten und war nur von kurzer Daner. (Bei Kröten wurden derartige Versuche nicht angestellt). Der Tetanus der Strecker war in allen Fällen ein langanhaltender, bochgradig tonischer. M u sk el gifte schädigen die Beuger des Unterschenkels und die Vastusgruppe des Oberschenkels eher als die Strecker und die Bicepsgruppe (Nenmann $)^{1}$ ). Eine Betheiligung der Muskeln selbst bei unserer Erscheinung anzunehmen, hätten diese Beobachtungen nicht genügt, $\mathrm{da}$ ja bei ihnen allen die Nerven nicht ausgeschaltet waren, wenn es nicht gelungen wäre, auch nach Ausschaltung des Nerven und seiner Endigungen das Ritter-Rollett'sche Phaenomen zu erzengen. Es ist dies gelungen. Griitzner ${ }^{2}$ ) hat durch Einspritzen von Wasser in die Aorta tief curarisirter Frösche unsere Erscheinung verschiedentlich beobachtet.

Um mit direkter elektrischer Reizung zu demselben Ziele zu gelangen, musste vor allen Dingen für ein ganz gleichmässiges Durchströmen des Schenkels mit dem elektrischen Strome gesorgt werden, was weder bei direkter Application der Electroden an die einzelnen Muskeln, noch bei Durchströmen des ganzen Schenkel, wenn man oben die eine, an den Zehen die andere Electrode anbringt, infolge der verschieden grossen Querschnitte des Schenkels der Fall ist. Ich bediente mich, um diesen Zweek zu erreichen, auf Vorschlag des Herrn Prof. Grützner des folgenden empfehlenswerthen Verfahrens: Ein kleines Kästchen aus Hartgummi ron geringer Tiefe und solcher Grösse, dass der Unterschenkel eines Frosches (resp. einer Kröte) frei darin sich nach allen Richtungen bewegen konnte, wurde mit physiologischer Kochsalzlösung gefiullt, und in dasselbe der Strom eines kleinen $\mathrm{Du}$ chenne'schen Inductionsapparates, welchen 2 (resp. 1) GroveElemente versorgten, mittelst breiter unpolarisirbarer Elektroden geleitet. In dieses elektrische Bad wurde der von der Haut befreite Unterschenkel einer tief curarisirten Kröte (bei elektrischer Reizung des Ischiadicus konnte nicht die geringste Zuckung mehr

1) Op. cit. pag. 32 etc.

2) Breslauer ärztliche Zeitschrift 1883, pag. 257. 
hervorgerufen werden) hineingelegt. Es trat, so lange die Messinghülse die Eisenstäbe des Apparates noch ganz umbüllte, eine sehr starke andauernde Beugnng ein, welehe allmählich beim Heransziehen der Messinghülse und der dadurch bedingten Stromverstärkung in Streckung überging. Am schönsten zeigte sich, wie bei indirekter Reizung so auch hier, das Phaenomen bei der Kröte, doch gelang auch beim Frosch der Nachweis desselben deutlich. Es genügte hier schon der Strom eines Grove-Elementes. Die Versuche, die anf diese Weise angestellt wurden, gelangen ausnahmslos aufs beste.

Nach diesen Ergebnissen der direkten Reizung kann kein Zweifel dariiber obwalten, dass bei un serer Erscheinung phy. siologisch verschiedene Muskelgruppen mit betheiligt sind, die der Beuger, welche schon anf schwächere, die der Strecker, welche erst auf stärkere Reize reagiren. Das gleiche wird dër Fall sein bei der Krebsscheere, den Verengerern und Erweiterern der Glottis und bei den vom Facialis versorgten Muskeln.

Was die Betheiligung der diese Muskeln versorgenden Nervenapparate anlangt, so ist es wahrseheinlich, dass auch sie Verschiedenheiten in der Erregbarkeit anfweisen. Es wäre natiirlich, dass die durch ihre Empfindlichkeit ausgezeichneten Beuger anch durch empfindlichere Nervenbahnen und Endapparate die Impulse zu ihrem Wirken erbielten. Einen Anbaltspunkt hierfür, wenigstens für Nervenendigungen, bietet der Umstand, dass bei Fröschen dureh Vergiftung mit Curare, welehes die Endapparate lähmt, ebenfalls eher die den Beugern analoge Vastusgruppe auf Nervenreize zu antworten aufhört als die den Streckern entsprechende Bicepsgruppe (wie Frl. Neumann gefunden hat ${ }^{1}$ ). Ich selbst habe auch Versuche mit Curare angestellt und fand, dass die Frösche schon nach verhältnissmässig kurzer Zeit (je nach der Stärke der verwendeten Dosis) nicht mehr im Stande waren, die Unterschenkel so vollkommen an sich zu ziehen, wie es gesunde thun, wenn man z. B. die Zehen mit verdünnter Essigsäure betupft. Es geschieht dies vielmehr zitternd und unvollständig. Dabei ist der Frosch noch wobl fähig zu springen, sogar noch recht weit, wenn auch etwas ungeschickt. Tödtet

1) Op. cit. pag. 31. 
man jetzt den Frosch und reizt schnell die Ischiadici, so kann man, wenn überhaupt noch Erregbarkeit auf elektrische Reize vorhanden, - was häufig nicht der Fall ist, trotzdem die vom Grosshirn ausgehenden Willensimpulse noch wirksam waren - nur noch Streckungen, keine Spur von Beugungen hervorrufen. Es zeigen also auch die Beuger und Strecker das gleiche Verbalten wie die Vastus- and Bicepsgruppe.

Der Gedanke liegt hier nahe, auch den anatomischen Bau der Beuge- und Streckmuskeln näher zu untersuchen, namentlich auf ihren Gehalt an dünnen und dicken Fasern. Die dicken Fasern sind die schnellen, leichter erregbaren, die dünnen die langsamen, ausdauernden, aber schwerer erregbaren, wie B o n b ö f f e r unter Grïtzner's Leitung gefunden hat ${ }^{1}$ ). Er sah bei Zählungen, dass beim Vastus des Frosches die dicken Fasern vorherrschen, beim Biceps dagegen dünne und dicke Fasern in nahezu gleichem Verhältniss vertreten waren ${ }^{2}$ ), was unserer Voraussetzung entsprecien würde. Für Beuger und Strecker des Unterschenkels existiren derartige Zählungen noch nicht. G. Schwalbe und Reitaro Mayed $\mathbf{a}^{3}$ ) baben eingehende und sorgfältige Untersuchungen über die Kaliberverhältnisse der Muskelfasern der verschiedensten Thiere und des Menschen angestellt. In der Arbeit des letzteren (pag. 138) heisst es: „Es liegt nahe, daran zu denken, dass auch die Beuger und Strecker der Extremitäten eine verschiedene Kalibercurve aufweisen möchten. Ich habe jedoch in den vorliegenden Beobachtungen keinen genügenden Anhalt gefunden." Jedenfalls sind die Untersuchungen üher die Frage, ob nicht doch anatomische Verschiedenheiten der Beuge- und Streckmuskeln vorhanden sind, noch nicht abgeschlossen.

Es hat sich also, um zum Schluss eine kurze Zusammenstellung zu geben, bei meinen Versuchen folgendes herausgestellt:

1) Ueber physiolog. Eigenschaften dünn- und dickfaseriger Muskeln bei Amphibien. Pflüger's Archiv Band 47 .

2) Op. cit. pag. 129.

3) Reitaro Mayeda, Ueber die Kaliberverhältnisse der quergestreiften Muskelfasern. Zeitschrift für Biclogie Band 27, pag. 119. G. Schwalbe und Reitaro Mayeda, Ueber die Kaliberverhältnisse der quergestreiften Muskelfasern des Menschen. Desgl. pag. 482. 
I. Das Ritter-Rollett'sche Phaenomen ist sow oh l beim Froseh (Rana temporaria und esculenta) als a u c h bei der Kröte (Bufo vulgaris) ni e ht allein $\mathrm{dur}$ eh elektrische, sondernauch chemische und meehanische Reizung àes Nerven deutlich hervorzurufen.

II. Da es gelungen ist, das Ritter-Rollett'sehe Phaenomen sowohl mittelst ehemischer als elektrischer direkter Muskelreizung hervorzurufen, so ist dieses Phaenomen keineswegs aussehliesslich auf verscbiedene Erregbarkeitsverhältnisseder Nervenfasern oder ibrerEndigungen zur üekzuführen, sondern beruht auch a nf der physiologischen Versehiedenheit der Muskelgruppender Beuger und Strecker. Es handelt sich also mit grösster Wahrscheinlichkeit um eine $p$ hysiologischeVersehiedenheit der gesammten betreffenden Nerv-Muskel-A pparate.

\section{Die pupillenerweiternden Nerven der Katze.}

Von

F. Nawrocki und J. Przybylski

in Warschau.

in Odessa.

Parfour da Petit) sah nach einseitiger Durchschneidung des Vagosympathicus bei Hunden Verengerung der entsprechenden Pupille. Petit's Versuche wurden mit demselben Erfolge zuerst durch Molinelli²) wiederholt, der anstatt der Durchschneidung die Unterbindung anwandte.

Durch Reid und Arnold ${ }^{8}$ ) wurde mit Sicherheit bekannt,

1) Citirt nach J. Budge, Ueber die Bewegung der Iris. Braunschweig 1854. S. 105.

2) Budge 1. c. S. 106.

3) Budge 1. c. S. 106. 